\title{
Modeling of Subsonic Cavity Flows by Neural Networks*
}

\author{
Mehmet Önder Efe ${ }^{\dagger}$ Marco Debiasi $;$ Hitay Özbay ${ }^{\S}$ and Mohammad Samimy
}

\begin{abstract}
Influencing the behavior of a flow field is a core issue as its improvement can yield significant increase of the efficiency and performance of fluidic systems. On the other hand, the tools of classical control systems theory are not directly applicable to processes displaying spatial continuity as in fluid flows. The cavity flow is a good example of this and a recent research focus in aerospace science is its modeling and control. The objective is to develop a $\mathrm{f}$ nite dimensional representative model for the system with appropriately defined inputs and outputs. Towards the goal of reconstructing the pressure fluctuations measured at the cavity floor, this paper demonstrates that given some history of inputs and outputs, a neural network based feedforward model can be developed such that the response of the neural network matches the measured response. The advantages of using such a model are the representational simplicity of the model, structural flexibility to enable controller design and the ability to store information in an interconnected structure.
\end{abstract}

\section{Introduction}

The fundamental objective of aerodynamic flow control is to develop strategies to excite the flow field so that a desired behavior is observed or some undesired phenomena are eliminated. Reducing the skin friction on the body of an aircraft constitutes a good example of control that results in reduced drag and thus reduced fuel consumption. Another example is the reduction of undesired high-level pressure fluctuations in a modern aircraft weapon bay, which cause material fatigue and damage to stores. The latter

*This work was supported in part by AFRL/VA and AFOSR under contract no F33615-01-2-3154.

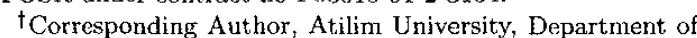
Mechatronics Engineering, Incek, Gölbaşı, TR-06836 Ankara, Turkey, E-mail: onderefe@ieee.org, Phone: +90-312-586-8358, Fax: +90-312-586-8091

$\ddagger$ Department of Mechanical Engineering, The Ohio State University, Columbus OH 43210, U.S.A.

$\S$ Department of Electrical and Electronics Engineering, Bilkent University, Bilkent, TR-06800, Ankara, Thrkey; on leave from Department of Electrical Engineering, The Ohio State University, Columbus, OH 43210, U.S.A., E-mail ozbay@ee.eng.ohio-state.edu

Department of Mechanical Engineering: The Ohio State University, Columbus OH 43210, U.S.A. phenomenon is known as cavity flow [1] and represents a good study benchmark due to the dynamical richness of its flow physics while its relevance to the above mentioned applications makes it an appealing subject for investigation. The challenges faced in controlling these flow systems clearly stipulate the development of well-interacting control media such that the existing literature is expanded as well as new application domains are initiated.

The control of aerodynamic flows has traditionally been accomplished by using passive techniques, and recently open loop strategies [2], which lack the advantages of feedback control schemes. More recents efforts have closed the loop based on ad hoc and trial and error type schemes, $[3,4,5,6]$. To make the approach more general, the loop must be closed based on flow models, which is a challenge due to the flow systems' infinite dimensionality, the complexity introduced by Navier-Stokes equations, the measurcment difficulties and the dynamics introduced by measurement and actuation devices. Extensive work have been done in the modeling of aerodynamic flows, $[7,8]$. The Proper Orthogonal Decomposition (POD) has constituted a widely used method for model development yet the POD based models have revealed convergence and/or drift problems in the behavior of temporal variables. Another approach is to exploit the physics of the problem. Rowley et al. and Williams et al. focused on this from a linear systems point of view, $[4,9,10]$. The acoustic waves produced by the shear-layer scattering at the downstream wall of the cavity reach, after some propagation delay time, the incoming flow in the receptivity region (i.e. the cavity upstream wall) and a delay-based coupled dynamics arise. The cited studies demonstrate that the shear layer development, acoustic scattering, cavity acoustics and receptivity can be represented dynamically as transfer functions. It must be noted that the parameters of the devised transfer function are tuned such that the frequency content of the experimental data obtained matches the frequency response of the model. For the model discussed in $[4,9,10]$, Yuan et al. [6] demonstrate the design and implementation of a $\mathcal{H}_{\infty}$ controller. A very good review of flow control literature is presented by Cattafesta et al., [11], in which the techniques and the advances are summarized from both a fluid mechanical point 
of view and a control specialist's point of view, and a tabulated comparison of operating conditions and obtained results is presented. A common starting point in all flow control applications is to describe the dynamics in terms of the mathematical tools; i.e. to devise a model capturing the essential dynamics. In this paper, the emphasis is on the reconstruction of the pressure measured from a particular location in the cavity based on the past observations from the same location and the excitation values.

The research carried out at Collaborative Center of Control Science at The Ohio State University has yielded a well-designed experimental facility, which provides an excellent platform for data acquisition and exploring the physics of flow phenomena and handling the control problem. The experimental setup, which is located at the Gas Dynamics and Turbulence Laboratory, is a small wind tumnel that provides continuous subsonic flow from very low speed to Mach 1 with a rich parameter space suitable for feedback control development. A shallow cavity with length to depth aspect ratio $L / D=4$ is recessed in the floor of the test section. The control is provided by a synthetic jet exhausting from a slot spanning the width of the cavity upstream wall. For a detailed information describing the simulations and experimental work on the cavity setup, the reader is referred to the past work of the authors in $[12,13]$.

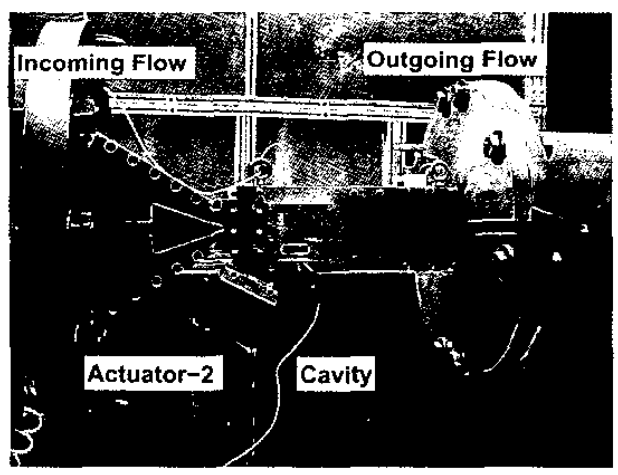

Figure 1: A general view of the experimental facility.

In Figure 1, the test section and its peripherals are shown. The incoming flow is directed towards the test section through a converging nozzle and leaves the test section by passing through an exhaust tube. A closer look at the test section setup is given in Figure 2, in which the entry slot of the control jet is visible at the receptivity region. The physics of the process should now be more clear. The input to the system is the signal applied to the actuator $(u(t))$, and the output is the pressure measured at

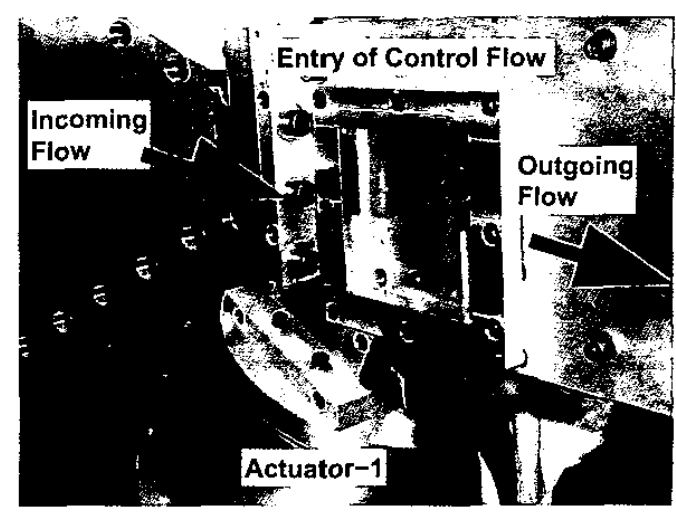

Figure 2: A closer look at the test section.

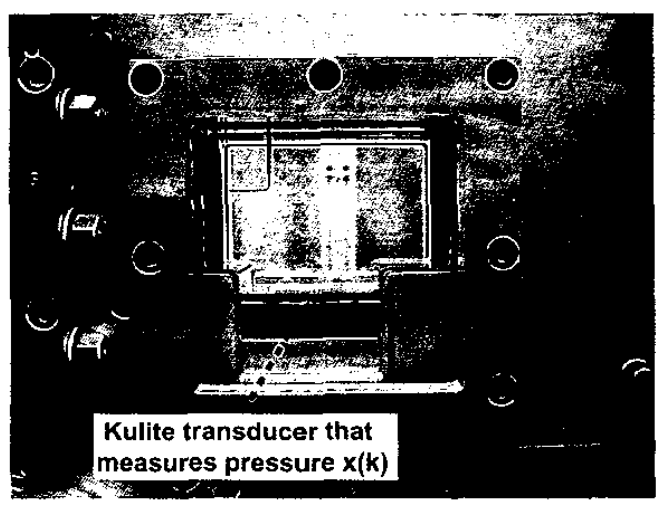

Figure 3: A closer look at the cavity floor.

the bottom of the cavity $(x(t))$. The Kulite dynamic pressure transducer located at the center of the cavity floor and used to observe the system can be seen in Figure 3. The same figure provides also a better view of the entry slot of the control flow as well as an appreciation of the cavity geometry. The cavity floor Kulite, as well as others at selected locations of the cavity domain, are connected to a Nationl Instrument data acquisition system hosted in a computer. In Figure 4, we illustrate a complete view of the cavity flow identification and control system. The contribution of this paper is to emphasize that the use of neural networks can offer promisingly flexible and accurate solutions.

This paper is organized as follows. The second section describes the neuroidentifier, the training algorithm, and the motivating factors. The identification results and specific implementation issues are 


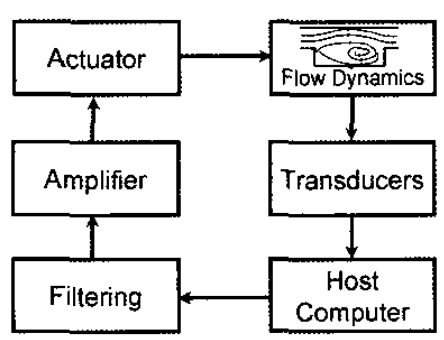

Figure 4: A complete view of the flow identification and control loop.

discussed in the third section. The conclusions are presented at the end of the paper.

\section{Neural Model}

In flow control applications, several factors encourage the use of neural networks. First of all, the process dynamics are quite complicated. Strong interdependencies betwcen the variables involved make it challenging to come up with a compact and useful representative model as shown by Samimy et al. $[12,13]$. Secondly, the system and its peripherals could be modeled individually, which is an inevitable stage in $[6,4,9]$. Finally, the data contains measurement noise. From this point of view, one sees that the neurocomputing algorithms and their connectionist models are excellent candidates for building a dynamical model containing the effects of process subsystems, which are the process itself, sensors, actuator and the filter(s) collectively. It is clear that the conditions above force the use of a model structure that is able to generalize the data while improving the information content progressively. A good deal of information about neural networks can be found in $[14,15]$ and the references therein.

An important issue in using neural networks is the parameter adjustment strategy. In the literature, many alternatives are proposed but the LevenbergMarquartdt technique is widely accepted as one of the most powerful ones. The algorithm is an approximation to the Newton's method, and both of them have been designed to solve the nonlinear least squares problem $[15,16]$. Consider a neural network having single output, and $N$ adjustable parameters (weights and biases) denoted by the vector $\underline{\omega}_{p}:=\left(\omega_{1 p}, \omega_{2 p}, \ldots, \omega_{N p}\right)$, where a subscript $p$ denotes the observation instant. If there are $P$ patterns in the ensemble, over which the interpolation is to be performed, a cost function qualifying the performance of the interpolation can be defined as

$$
E:=\frac{1}{2} \sum_{p=1}^{P}\left(d_{p}-x_{p}\left(\underline{\omega}_{p}\right)\right)^{2},
$$

where $d_{p}$ and $x_{p}$ are the desired and observed responses at index $p$, respectively. It should be noted that $x_{p}$ is a function of $\underline{\omega}_{p}$. The parameter update law based on Levenberg-Marquardt optimization is performed as

$$
\begin{aligned}
\underline{\omega}_{k+1}= & \underline{\omega}_{k}-\left(\mu I_{N \times N}+J\left(\underline{\omega}_{k}\right)^{\mathrm{T}} J\left(\underline{\omega}_{k}\right)\right)^{-1} \times \\
& J\left(\underline{\omega}_{k}\right)^{\mathrm{T}} \underline{e}\left(\underline{\omega}_{k}\right),
\end{aligned}
$$

where $J$ is the Jacobian, whose $p^{\text {th }}$ row is composed of $\left(\frac{\partial e_{p}\left(\underline{\omega}_{p}\right)}{\partial \omega_{1 p}}, \frac{\partial e_{p}\left(\underline{\omega}_{p}\right)}{\partial \omega_{2 p}}, \ldots, \frac{\partial e_{p}\left(\underline{\omega}_{p}\right)}{\partial \omega_{N p}}\right)$ with $e_{p}\left(\underline{\omega}_{p}\right):=d_{p}-$ $x_{p}\left(\underline{\omega}_{p}\right)$. After a suitable implementation of the training algorithm, one can come up with a model having the structure

$$
\begin{aligned}
x_{k+1}= & f\left(x_{k}, x_{k-1}, \ldots, x_{k-n},\right. \\
& \left.u_{k}, u_{k-1}, \ldots, u_{k-m}\right),
\end{aligned}
$$

where the delay depths $n$ and $m$ are user specified parameters. The process of learning refines the function $f$ and the resulting connectionist structure can rebuild the flow measurements so that the quantity in (1) is minimized over the set of $P$ training pairs.

\section{Identification of the Flow}

The identification mechanism of a flow system by a neural network is depicted in Figure 5, where the neural network is forced to imitate the response of the system under the same operating conditions. The training algorithm refines the input-output description of the neural identifier so that the estimation error is minimized over a set of representative inputoutput data.

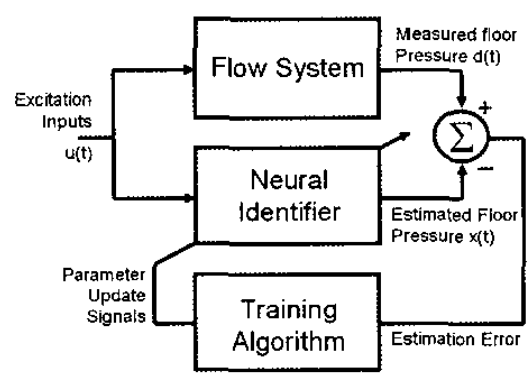

Figure 5: Identification of a system. 
The first stage of the identification process is to collect the data. For this purpose, we initially focus our attention to a Mach 0.3 flow. We acquired two extensive sets of data from the pressure transducers in the test section by sampling the corresponding amplified and anti-aliasing filtered signals at a rate of $200 \mathrm{kHz}$. The actuator excitation signal was highpass filtered to stop any spectral content below $1 \mathrm{kHz}$ which would damage the actuator. Of the two sets of data collected, one is used for training while the other is used to validate the model. For this purpose, the training data set has been acquired when a $2 \mathrm{kHz}$ sinusoidal excitation voltage of $4 V_{r m s}$ is sent to the actuation device. The pressure is read from the Kulite transducer seen in Figure 3. During the modeling trials, we have chosen a feedforward neural network with a single hidden layer. The neurons in the hidden layer contain the hyperbolic tangent nonlinearity and the output neurons are chosen to be linear. The input vector to the neuroidentifier is composed of $\left[u_{k}, u_{k-1}, x_{k}, x_{k-1}, x_{k-2}\right]$, i.e. $n=2$ and $m=1$, and the response of the network to this vector is $x_{k+1}$. Furthermore, we have used 12 hidden neurons letting us ond up with 5-12-1 configuration. With the Levenberg-Marquardt scheme, the entire data set, which contains 16381 pairs, passed through the neural network 50 times (epoches) while modifying the weights and biases according to (2). At the end of this process, the Mean Squared Error (MSE) has decreased below $8.85 \mathrm{e}-6$.

The tests have been carried out with another sinusoidal input signal with amplitude $4 \mathrm{~V}$ and frequency $3.25 \mathrm{kHz}$, and the output from the process and the response of the neural network have been recorded. According to the acquired data, the results scen in Figure 6 and 7 have been obtained.

According to the results illustrated in the top row of Figure 6, the two curves are almost indistinguishable. The discrepancy curve shown in the bottom row confirms the accurate reconstruction claim of the paper. The comparison in the frequency domain is presented in Figure 7, where the two curves are similar enough to use the devised model as a dynamical representative. A look at the error magnitude in the bottom row of Figure 7 emphasizes the reconstruction accuracy of the neuroidentifier. It is visible that the error curve has a reasonably small magnitude over a wide range of frequencies.

It should be noted that the topology of the neural network and the type of nonlinearities absolutely depend upon the designer's perceptions, intuitions and experience. There is not an analytical way to determine the number of hidden layers as well as the number of neurons. Based on our extensive work, the simplest configuration yielding a satisfactorily precise response is the one presented here.
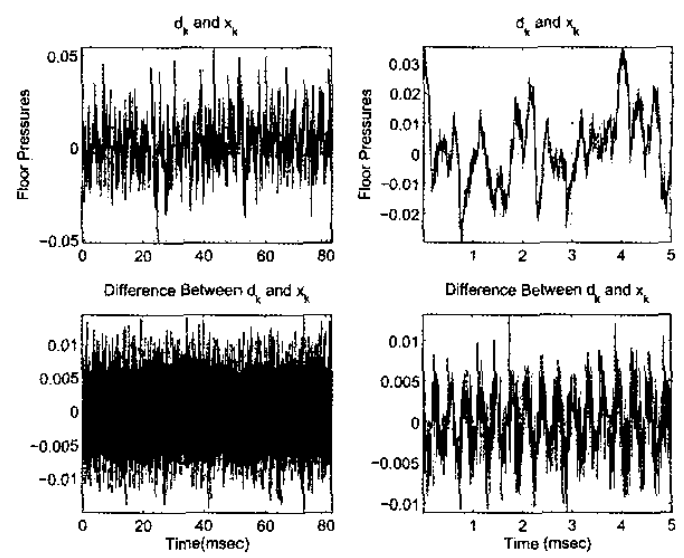

Figure 6: Top row: The data obtained from the Kulite transducer $\left(d_{k}\right)$ and the response of the neuroidentifier $\left(x_{k}\right)$. Bottom row: The estimation error, $d_{k}-x_{k}$. The plots on the left are for the entire test set while the plots on the right are only for the first 1000 samples i.e. $5 \mathrm{~ms}$ time.

A last issue that should be discussed is a comparison of the findings with those appeared in the literature before.

a) POD is computationally intensive, vulnerable to small numerical imprecision and the models may exhibit progressively increasing drift and/or instability. Aside from all these, POD models for flow problems are generally very complicated. Another difficulty of POD based flow models is the process of spatial separation of the control entry. The advantage, on the other hand, is that, once it works, the model is global over the entire cavity domain and the individual performance metrics can be distributed over the physical geometry of the cavity.

b) Delay based models as studied in $[8,4,9,10]$ are very useful in terms of designing controllers, [6], yet it is difficult to inject the dynamical properties of sensors, actuators, and filters in a collective manner. The match in frequency domain can be achieved to some extent but the time domain match is not as good as the one we present here. The advantage of using these models is the possibility of using the tools of linear control systems theory.

c) Neural network based identification scheme is superior to the ones mentioned above in the sense that it is based on the data containing the effects of system components collectively. The designer can decide on the topology of the network as well as the training scheme to update the parameters. The trained network can be used for feedback controller design, [17]. 

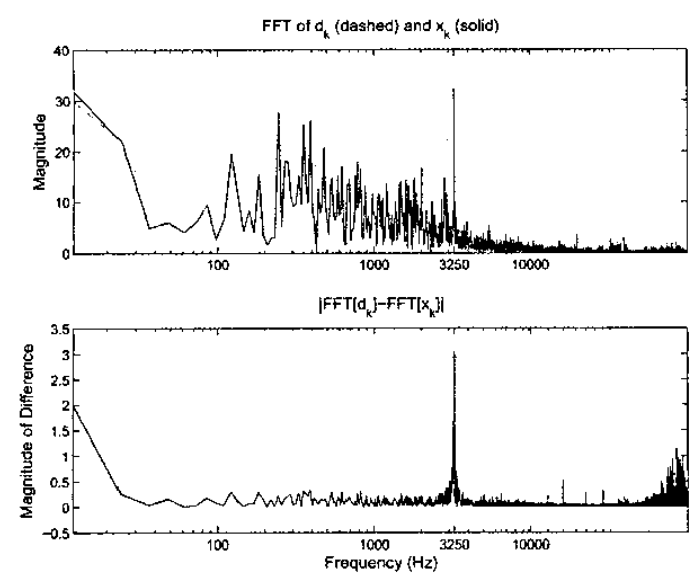

Figure 7: Top row depicts the FFT magnitude pictures of the desired $\left(d_{k}\right)$ and the estimated $\left(x_{k}\right)$ signals. The bottom plot illustrates the quantity $\left|\operatorname{FFT}\left(d_{k}\right)-\operatorname{FFT}\left(x_{k}\right)\right|$.

\section{Conclusions}

In this paper, identification of cavity flow system is studied. A neural network is used to imitate the behavior of the process under investigation. Several past values of the output and the input are fed to the neuroidentifier and the likely next, output is estimated accurately. This is done by suitably tuning the adjustable parameters of the neural network by utilizing the Levenberg-Marquardt optimization technique. The results are quite promising for devising a feedback control scheme to manage the behavior of the flow field.

\section{Acknowledgments}

This work was supported in part by the AFRL/VA and AFOSR through the Collaborative Center of Control Science at the Ohio State University (Contract F33615-01-2-3154).

The authors would like to thank Dr. J.H. Myatt, Dr. J. DeBonis, Dr. R.C. Camphouse, X. Yuan, E. Caraballo and P. Yan for fruitful discussions in devising the presented work.

\section{References}

[1] Rossiter, J.E., "Wind Tunnel Experiments on the Flow Over Rectangular Cavities at Subsonic and Transonic Speeds," RAE Tech. Rep. 64037, 1964, and Aeronautical Research Council Reports and Memoranda No. 3438, October 1964.
[2] Gad-el-Hak, M., Flow Control - Passive, Active, and Reactive Flow Management, Cambridge University Press, New York, NY, 2000.

[3] Cattafesta, L.N. III, Garg, S., Choudhari, M., and Li, F., "Active Control of Flow-Induced Cavity Resonance," U.S.A. 1997 (Paper: AIAA97-1804).

[4] Williams D.R., Rowley C.W., Colonius, T., Murray, R.M., MacMartin, D.G., Fabris, D., Albertson, J., "Model Based Control of Cavity Oscillations Part I: Experiments," 40th Aerospace Sciences Meeting, Reno, NV, U.S.A., 2002 (Paper: AIAA2002-0971).

[5] Debiasi, M. and Samimy, M., "An Experimental Study of the Cavity Flow for Close-Loop Flow Control," 33rd AIAA Fluid Dynamics Conference and Exhibits, June 23-26, Orlando, Florida, U.S.A., 2003 (Paper: AIAA2003-4003).

[6] Yuan, X., Efe, M.Ö., and Özbay, H., "On Delay-Based Linear Models and Robust Control of Cavity Flows," presented at the NSF-CNRS Workshop on Advances in Time Delay Systems, Jan. 22-24, France, 2003.

[7] Ravindran S.S., "A Reduced Order Approach for Optimal Control of Fluids Using Proper Orthogonal Decomposition," Int. J. for Numerical Methods in Fluids, v.34, pp.425-488, 2000.

[8] Rowley, C.W., Modeling, Simulation and Control of Cavity Flow Oscillations, Ph.D. Thesis, California Institute of Technology, Pasadena, U.S.A., 2001

[9] Rowley, C.W., Williams, D.R., Colonius, T., Murray, R.M., MacMartin, D.G., Fabris, D., "Model Based Control of Cavity Oscillations Part II: System Identification and Analysis:" 40th Aerospace Sciences Meeting, Reno, NV, U.S.A., 2002, (Paper: AIAA2002-0972).

[10] Rowley, C.W., Colonius, T., Murray, R.M., "Dynamical Models for Control of Cavity Oscillations," 7th AIAA/CEAS Aeroacoustics Conf., May 28-30, Maastricht, The Netherlands, 2001, (Paper: AIAA2001-2126)

[11] Cattafesta, L., Williams, D., Rowley, C.W. and Alvi, F., "Review of Active Control of Flow-Induced Cavity Resonance," 33rd AIAA Fluid Dynamics Conference and Exhibits, June 23-26, Orlando, Florida, U.S.A., 2003 (Paper: AIAA2003-3567).

[12] Samimy, M., Debiasi, M., Caraballo, E., Özbay, H., Efe, M. Ö., Yuan, X., DeBonis, J., and Myatt, J.H., "Closed-Loop Active Flow Control - A 
Collaborative Approach:" 41st AIAA Aerospace Sciences Meeting and Exhibit, Jan. 6-9, Reno, Nevada, U.S.A., 2003 (Paper: AIAA2003-0058).

[13] Samimy, M., Debiasi, M., Caraballo, E., Özbay, H., Efe, M. Ö., Yuan, X., Myatt, J.H. and DeBonis, J., "Development of Closed-Loop Active Flow Control Based on Low Dimensional Model," 33rd AIAA Fluid Dynamics Conference and Exhibits, June 23-26, Orlando, Florida, U.S.A., 2003 (Paper: AIAA2003-4258).

[14] Jang, J.-S.R., Sun, C.-T., Mizutani, E., NeuroFuzzy and Soft Computing, PTR Prentice-Hall, 1997.

[15] Haykin, S., Neural Networks, Macmillan College Printing Company, New Jersey, 1994.

[16] Hagan, M.T. and Menhaj, M.B., "Training Feedforward Networks with the Marquardt Algorithm", IEEE Trans. on Neural Networks, v.5, No. 6, pp. 989-993, November 1994.

[17] Narendra, K.S. and Parthasarathy, K., "Identification and Control of Dynamical Systems Using Neural Networks", IEEE Trans. on Neural Networks, v.1, No. 1, pp. 4-27, 1990. 